\title{
Ultra-pure, water-dispersed Au nanoparticles produced by femtosecond laser ablation and fragmentation
}

This article was published in the following Dove Press journal:

International Journal of Nanomedicine

18 July 2013

Number of times this article has been viewed

\author{
Reda Kubiliūtè',2 \\ Ksenia A Maximova ${ }^{3}$ \\ Alireza Lajevardipour ${ }^{1}$ \\ Jiawey Yong' \\ Jennifer S Hartley' \\ Abu SM Mohsin' \\ Pierre Blandin ${ }^{3}$ \\ James WM Chon' \\ Marc Sentis ${ }^{3}$ \\ Paul R Stoddart ${ }^{\prime}$ \\ Andrei Kabashin ${ }^{3}$ \\ Ričardas Rotomskis ${ }^{2}$ \\ Andrew HA Clayton ${ }^{1,4}$ \\ Saulius Juodkazis ${ }^{1,4}$ \\ 'Centre for Micro-Photonics \\ and Industrial Research Institute \\ Swinburne, Faculty of Engineering \\ and Industrial Sciences Swinburne \\ University of Technology, Hawthorn, \\ VIC, Australia; ${ }^{2}$ Laboratory of \\ Biomedical Physics, Vilnius University \\ Institute of Oncology, Baublio, Vilnius, \\ Lithuania; ${ }^{3}$ Aix-Marseille University, \\ Centre National de la Recherche \\ Scientifique (CNRS), Lasers, Plasmas \\ and Photonics Processing Laboratory, \\ Campus de Luminy, Marseille, France; \\ ${ }^{4}$ The Australian National Fabrication \\ Facility, Victoria node, Faculty of \\ Engineering and Industrial Sciences, \\ Swinburne University of Technology, \\ Hawthorn, VIC, Australia
}

Correspondence: AHA Clayton Centre for Micro-Photonics and Industrial Research Institute Swinburne, Faculty of Engineering and Industrial Sciences Swinburne University of Technology,

Hawthorn, VIC, 3122, Australia

$\mathrm{Tel}+61392145719$

Fax +61392145435

Email aclayton@swin.edu.au
Abstract: Aqueous solutions of ultra-pure gold nanoparticles have been prepared by methods of femtosecond laser ablation from a solid target and fragmentation from already formed colloids. Despite the absence of protecting ligands, the solutions could be (1) fairly stable and poly size-dispersed; or (2) very stable and monodispersed, for the two fabrication modalities, respectively. Fluorescence quenching behavior and its intricacies were revealed by fluorescence lifetime imaging microscopy in rhodamine $6 \mathrm{G}$ water solution. We show that surface-enhanced Raman scattering of rhodamine $6 \mathrm{G}$ on gold nanoparticles can be detected with high fidelity down to micromolar concentrations using the nanoparticles. Application potential of pure gold nanoparticles with polydispersed and nearly monodispersed size distributions are discussed.

Keywords: nanotechnologies applications, methods of nanofabrication and processing, materials for nanomedicine

\section{Introduction}

Due to unique optical properties associated with the excitation of plasmons, gold-based nanomaterials (nanoparticles, nanorods, core shells, and designed nanoparticle arrays) have become increasingly popular for tasks of biological sensing, imaging, and therapy. In particular, extremely efficient optical absorption/scattering, with typically $\sim 10^{5}$-fold higher cross-section than dyes, has been employed for the formation of optical contrast in biological imaging, ${ }^{1,2}$ as well as for light-induced hyperthermia of solid tumors. ${ }^{2-4}$ On the other hand, strong electric field enhancement near gold $(\mathrm{Au})$ nanoparticles is actively employed in surface-enhanced Raman spectroscopy (SERS) to detect and identify biological and chemical species of interest in in vitro ${ }^{5-7}$ and in vivo ${ }^{8}$ geometries. Finally, small $(<2 \mathrm{~nm})$ Au nanoparticles can exhibit strong fluorescence, making them good candidates for imaging applications. ${ }^{9} 10$ However, these applications are often complicated by potential toxicity issues, arising as a result of inadequate purification procedures of nanoparticles and nano-engineered materials obtained via synthetic pathways using toxic or non-biocompatible substances. ${ }^{11}$ As an example, standard chemical procedure for the fabrication of colloidal Au nanoparticles is based on the reduction of a precursor salt with a reducing agent, which inevitably leads to surface contamination, mainly by residual anions and the reducing agent, complicating the above stated applications, especially in vivo. ${ }^{12}$ The development of new methods for synthesizing plasmonic nanoparticles is an active field of research, ${ }^{13,14}$ including fabrication by short laser pulses. ${ }^{15,16}$ SERS applications are currently on the way to clinical trials, ${ }^{17}$ and generic properties on nanomaterials in antiviral and cancer treatment applications are under fundamental study and in vivo experiments. ${ }^{18,19}$ 
Pulsed laser ablation in liquids has recently emerged as a novel "green" tool for synthesis of colloidal nanomaterials, which offers a breakthrough in the solution of the toxicity problem. ${ }^{20}$ In this method, laser radiation is used to ablate a solid target in liquid ambience yielding to the production of nanoclusters, which are then released into the liquid, forming a colloidal nanoparticle solution. When ablated in a pure aqueous environment, the method enables contamination of the nanoparticle surface, which is typical for chemically prepared counterparts, to be avoided. Although the size distribution of gold nanoparticles prepared by conventional "long" (nanosecond) laser ablation in deionized water is typically very broadened, ${ }^{21-28}$ it can be much improved by adding reactive surfactants (eg, sodium dodecyl sulfate). ${ }^{23,24}$

The employment of femtosecond laser radiation is of particular interest for nanoparticle synthesis, as it cannot only provide superior and controllable size characteristics, ${ }^{29,30}$ but can also improve solution stability due to a negative charge of formed nanostructures yielding to electrostatic repulsion effects. ${ }^{31}$ Here, the employment of femtosecond fragmentation from preliminarily formed nanoparticles can further enhance advantages of ultrashort laser ablation to produce almost monodispersed gold nanoparticles with exceptional stability of colloidal solution in the absence of any protecting ligand. ${ }^{2,3}$ In addition, lasersynthesized nanoparticles can exhibit unique surface chemistry. In particular, due to a slight surface oxidation ${ }^{31}$ gold nanoparticles can react not only with non-biocompatible surfactants (sodium dodecyl sulfate and cetyl trimethyl ammonium bromide), but also with $\mathrm{OH}$ groups of fully biocompatible substances such as cyclodextrins ${ }^{32}$ or some polymers (eg, polyethylene glycol and dextran), ${ }^{33}$ opening up new attractive pathways for biological applications. Surprisingly, despite the above-stated set of formidable properties, optical characteristics of laser-synthesized nanoparticles (except the absorption/scattering spectral feature associated with the excitation of plasmons) have not yet been systematically studied.

This paper was conceived as an attempt to fill the existing gap in optical characterization of laser-synthesized nanoparticles. We first prepare aqueous solutions of unprotected Au nanoparticles by methods of femtosecond laser ablation and fragmentation, and then examine their fluorescence, Raman scattering, and fluorescence lifetime of dyes in the presence of pure Au nanoparticles. Numerical modeling of optical properties and comparison with experimental extinction spectrum as well as stability of solution were examined.

\section{Samples and methods Synthesis of Au nanoparticles}

For our tests, we prepared aqueous solutions of pure Au nanoparticles by two femtosecond laser ablation-based routes. The first protocol implied the involvement of a classical geometry of femtosecond laser ablation from a gold target in liquid ambience. ${ }^{29,30}$ Briefly, the gold target (99.99\%) was placed at the bottom of a glass vessel filled with $20 \mathrm{~mL}$ of highly deionized water $(18.2 \mathrm{M} \Omega \mathrm{cm}$ ). A $2.3 \mathrm{~mm}$ diameter beam of $\mathrm{Yb}: \mathrm{KGW}$ femtosecond laser (Amplitude Systems [Pessac, France], $1025 \mathrm{~nm}, 480 \mathrm{fs}, 500 \mu \mathrm{J}, 1-5 \mathrm{kHz}$ ) was focused with the help of a $750 \mathrm{~mm}$ lens onto the target surface to provide the ablation of material. The target was moved at a scanning velocity of $0.35 \mathrm{~mm} / \mathrm{s}$ in the focusing plane to obtain identical surface conditions during the laser ablation, while the thickness of the water layer above the target was about $1 \mathrm{~cm}$. Such ablation geometry normally leads to a reddish coloration of the aqueous solution (with some yellow tints in transmitted light) after 2-5 minutes of the experiment. A droplet of the prepared solution was then dropped onto a carbon-coated copper-based transmission electron microscopy (TEM) grid, dried, and then examined by TEM (see the Characterization of Au nanoparticles section for details). Laser synthesized nanoparticles had a broader size distribution ranging from a few to several tens of nanometers. Here, relatively large colloids were often interlinked by a large amount of smaller particles to form big aggregates in the solution. From statistical size analysis, the mean size and size dispersion were $15 \pm 10 \mathrm{~nm}$ at full width half-maximum (FWHM), as shown in Figure 1. It is noteworthy that numerical modeling of size distribution based only on the extinction spectra (see Results section), without consideration of the actual TEM size distribution, yielded the very same result.

As the second protocol, we employed methods of two step femtosecond laser fragmentation procedure introduced in a previous study. ${ }^{34}$ The $10 \mathrm{~mL}$ of nanoparticle solution prepared by the first protocol was transferred into a glass cuvette and irradiated, in the very center of the cuvette, by a focused laser beam of the $\mathrm{Yb}: \mathrm{KGW}$ femtosecond laser (using the same focusing lens), while the solution was stirred by a magnet to homogenize the ablation process. We used relatively low laser fluences $\left(1 \mathrm{~J} \cdot \mathrm{cm}^{2}\right)$ to avoid the phenomenon of laser-assisted plasma breakdown of the liquid, but the radiation intensity was high enough to ablate the suspended nanoparticles. As shown in previous studies, ${ }^{32-34}$ when the pumping laser wavelength is relatively far from the absorption band of $\mathrm{Au}$ nanoparticles (depending on nanoparticle size), the nanoparticles are predominantly ablated by radiation from a white light 


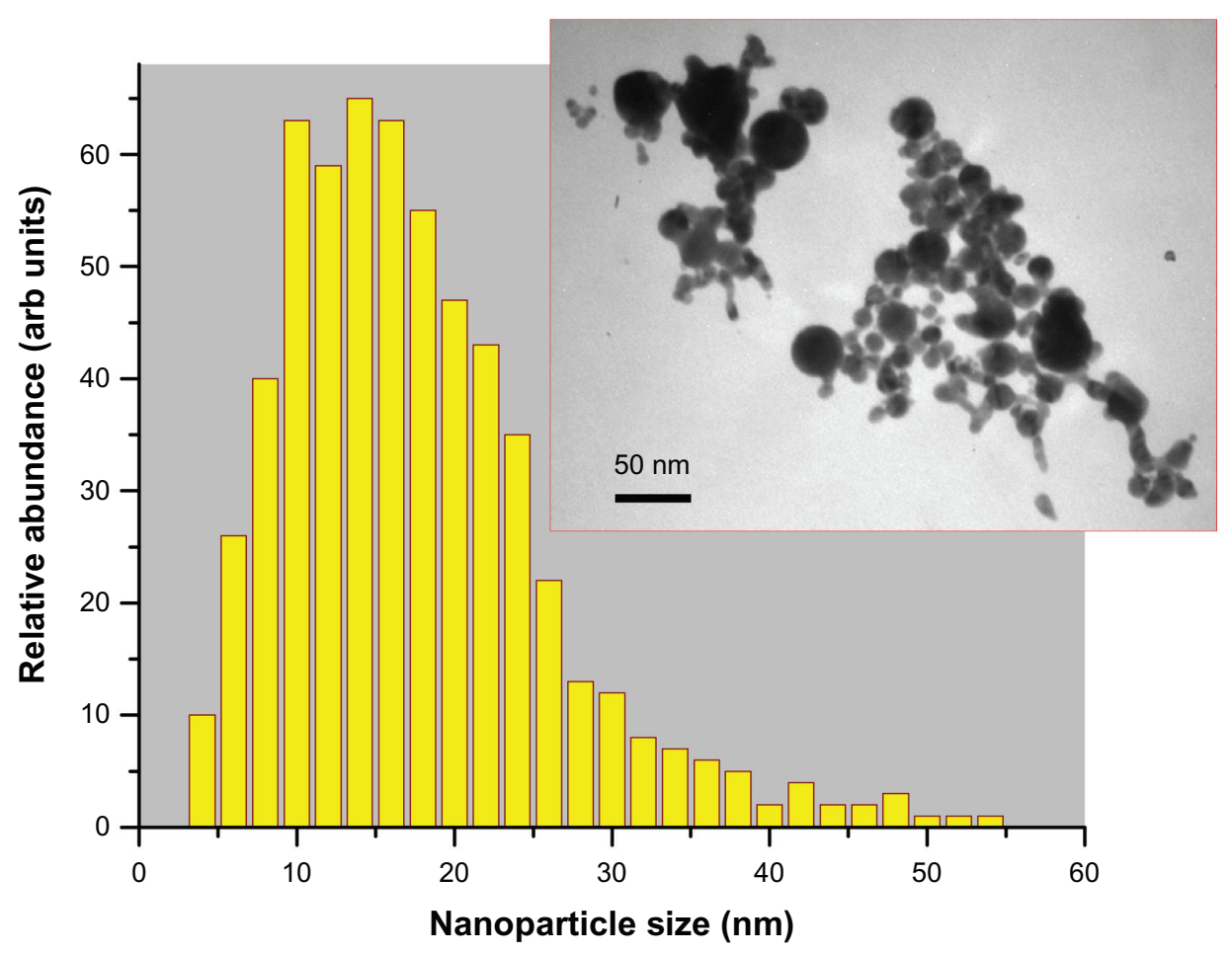

Figure I Size distribution of gold nanoparticles in the ablated sample solution (protocol I, sample Number I) determined by transmission electron microscopy image (inset) analysis: $15 \pm 10 \mathrm{~nm}$ (at full width half-maximum).

supercontinuum (presenting a wide spectrum from ultraviolet [UV] to infrared of femtosecond pulse length), generated in the water environment due to nonlinear self-focusing effects that normally accompany femtosecond laser-liquid interaction. ${ }^{37}$ In our experiments, this white light supercontinuum was clearly visible in the liquid volume. The femtosecond-laser fragmentation process led to a change of solution color into deep (dark) red without any sign of yellow tints. After 40 minutes of fragmentation, the nanoparticle solutions were extremely stable and did not show any sign of precipitation, even after months of storage at room temperature. As shown in Figure 2, the mean size of nanoparticles was about $24 \mathrm{~nm}$, while the size dispersion was very weak (less than $10 \mathrm{~nm}$ FWHM). To study solution stability effects, we carried out fragmentation for 20 minutes, which led to a smaller mean size (15-17 nm) and broader size dispersion (14 nm FWHM). Samples prepared by femtosecond-laser ablation from the Au target (protocol 1; Figure 1) are referred to as sample Number 1. Samples prepared by complete and partial femtosecond-laser fragmentation (protocol 2) are referred to as Number 2 (Figure 2) and Number 2a, respectively.

\section{Characterization of Au nanoparticles}

Absorbance spectra were measured with a Varian Cary Win UV spectrometer (Varian Inc, Melbourne, VIC, Australia) and fluorescence spectra with a Varian Cary Eclipse fluorometer (Varian Inc, Australia). Fluorescence excitation and emission slits were $5 \mathrm{~nm}$; measurements were taken in $1 \mathrm{~cm}$ path length quartz cells (Hellma, Müllheim, Germany).

Particle size in the solution-aging study was measured using dynamic light scattering at a $90^{\circ}$ scatter angle on a Nanoparticle Size Analyzer system (Brookhaven Instruments Corp, Holtsville, NY, USA). Data were acquired at $25^{\circ} \mathrm{C}$, and sample solutions were prepared by diluting $\mathrm{Au}$ nanoparticles into deionized water. The results are given as the mean value of 10 measurements. An ultrasonic revival of the aged colloidal solution was accomplished in a Bandelin Sonorex RK 156 BH (Bandelin Electronic GmbH, Berlin, Germany) commercial ultrasonic bath operating at $35 \mathrm{kHz}$ and with power of $215 \mathrm{~W}\left(8.6 \mathrm{~W} / \mathrm{cm}^{2}\right)$.

The Nikon Eclipse Ti microscope with a Lambert fluorescence lifetime imaging microscopy (FLIM) attachment was used to carry out experiments on the quenching of the rhodamine 6G (Rh6G) dye lifetime in the presence of gold nanoparticles.

TEM images of the colloidal nanoparticles were obtained with a JEOL (Tokyo, Japan) 1010 microscope operating at an accelerating voltage of $100 \mathrm{kV}$. TEM samples were prepared by dropping $20 \mu \mathrm{L}$ of the concentrated particle samples on 300 mesh carbon-coated TEM grids and dried in air. 


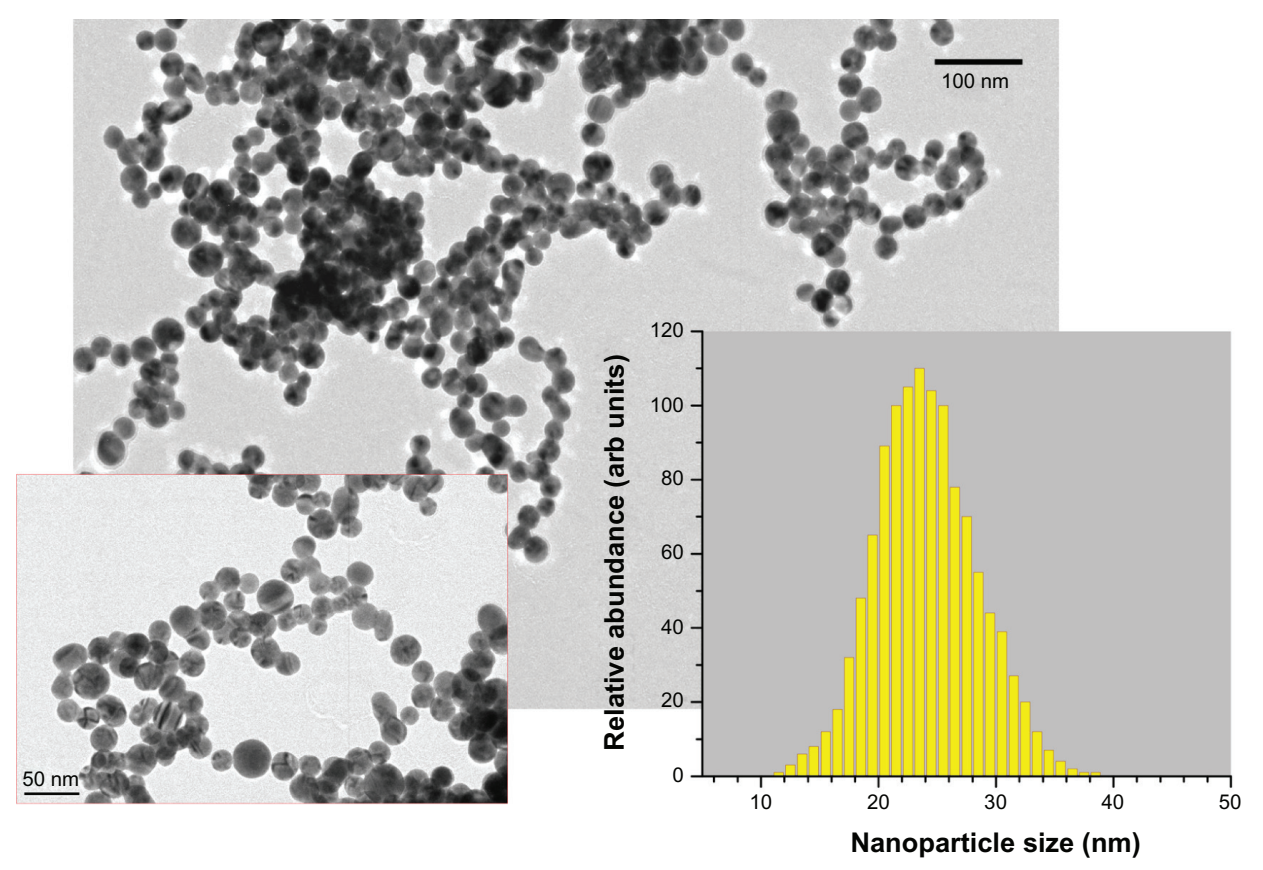

Figure 2 Transmission electron microscopy (TEM) images of nanoparticles from the most stable gold (Au) colloidal solution (protocol 2; sample Number 2) fragmented by femtosecond-laser irradiation in pure $\mathrm{pH}=7$ water (at different magnifications). Inset shows the size (diameter) distribution $24 \pm 5 \mathrm{~nm}$ using TEM image analysis.

\section{SERS samples}

SERS samples were prepared using a mercaptosilane cross linker to attach the gold nanoparticles to the oxidized surface of a silicon wafer. This was used to form an even layer of nanoparticles on the surface and prevent highly concentrated groupings, thus allowing reproducible measurements. ${ }^{38}$ The silicon wafer was sonicated for 10 minutes in detergent Decon 90 (Bacto Laboritories Pty, Ltd., Mt Pritchard, NSW, Australia), rinsed with deionized water and dried with pure nitrogen to ensure a clean surface prior to treatment. An oxide layer was then deposited onto the surface using an ozone ashing. The wafer was immersed in a $10 \mathrm{mM}$ solution of (3-mercaptopropyl) trimethoxysilane 95\% (Sigma-Aldrich, St Louis, MO, USA) in ethanol 99.5\% (Sigma-Aldrich) and allowed to functionalize overnight. The wafer was rinsed thoroughly with ethanol and dried with pure nitrogen prior to immersion in a solution of $\mathrm{Au}$ nanoparticles for 24 hours. The nanoparticles were sonicated for an hour before immersion to resuspend them. The sample was then soaked in a $4.3 \mu \mathrm{M}$ solution of $\mathrm{Rh} 6 \mathrm{G}$ in water for 15 minutes, after which it was rinsed with deionized water. SERS spectra were collected using a inVia Raman (Renishaw, Wotton-under-Edge, Gloucestershire, UK) microscope with an excitation wavelength of $632.8 \mathrm{~nm}$ and a holographic grating with 1800 lines $/ \mathrm{mm}$. The power exciting the sample was approximately $0.4 \mathrm{~mW}$. A $50 \times$ long working distance lens (numerical aperture $=0.5$ ) was used for excitation and collection of spectra. The spectra were collected with three exposures of 10 seconds each. Multiple spectra were taken over the sample to assess reproducibility. No agglomeration of colloidal nanoparticles was observed with an optical microscope at a resolution of $\sim 1 \mu \mathrm{m}$; if surface anchoring was not used, clustering of Au nanoparticles occurred and was discernable under optical observation. We show SERS data only for sample Number 1.

Spectra were analyzed with Matlab (Math Works, Natick, MA, USA). The multiple reference and Rh6G spectra were averaged. The averaged reference was subtracted from the averaged Rh6G spectrum. However, due to a sample-induced change in the Raman background, the reference spectrum was shifted and warped slightly to provide a close fit for the subtraction. The background-corrected Rh6G data was smoothed with a Savitzky-Golay filter using a window size of 15 and a third-order polynomial. This method of smoothing is suitable as it minimally affects the shape and height of the peaks yet significantly improves the signal-to-noise ratio. Origin Pro (Ozapin Lab, Northampton, MA, USA) was used to plot the final data. A background subtraction was implemented to remove any artifacts that appeared during subtraction. The peak fit function was used to find and label peaks.

\section{Results}

\section{Structural and optical characterization}

By controlling femtosecond-pulse energy in ablation and fragmentation conditions, fabrication of colloidal nanoparticles was optimized for solution stability. It was found that the most stable solution (months without sedimentation) was obtained at the most narrow size distribution of preferentially spherical 
Au nanoparticles. Figure 2 shows TEM images and size analysis of the most stable colloidal solution (sample Number 2); no agglomeration and sedimentation was observed over a period of 2 months in water without any surface treatment.

Figure 3 shows absorption, fluorescence, and extinction spectra of the stable nanoparticles. The narrow absorption peak is consistent with a narrow size distribution of preferentially spherical colloidal nanoparticles. The absorption shoulder at the shortest wavelengths is due to the smallest particles; the cross section of total losses, or extinction, which is due to scattering and absorption together scales as $\sigma_{e x} \equiv \sigma_{a b s}+\sigma_{s c} \alpha r^{3} / \lambda+r^{6} / \lambda^{4}$, where $r$ is the radius of the nanoparticle and $\lambda$ is the wavelength. When the particle size is comparable to the electron travel length during an optical cycle $V_{F} \tau_{s}=1-2 \mathrm{~nm}$, the spectral dependence saturates (the nonlocality limit), ${ }^{39}$ where $V_{F}$ is the Fermi velocity and $\tau_{s} \sim 3 \mathrm{fs}$. At $5.1 \mathrm{eV}(241 \mathrm{~nm})$, there is a peak in absorption, which should be related to the electron work function of gold.

Fluorescence and its excitation spectra are defined by the electron transition from the Fermi level to $d$-bands in gold. ${ }^{40}$ The nanoparticle fluorescence at $300 \mathrm{~nm}(4.1 \mathrm{eV})$ and $409 \mathrm{~nm}$ $(3 \mathrm{eV}$ ) are consistent with the three $d$-bands (one more band is at $526 \mathrm{~nm}$ or $\left.2.34 \mathrm{eV}^{40}\right)$. Strong emission is excited when $\mathrm{Au}$ is ionized at $5.1 \mathrm{eV}(241 \mathrm{~nm})$. Fluorescence at $410 \mathrm{~nm}$ was observed in the case of stable nanoparticles with the excitation maximum at $296 \mathrm{~nm}$ (see the excitation peak at 290-300 nm in Figure 3). This is consistent with the presence of the very small few-nanometer-diameter nanoparticles whose presence is also discernable as a plateau region in the optical extinction spectrum (at that particle size, the absorption becomes strong and spectrally featureless). Size distribution data from Figure 2 were used to model the absorption spectrum and close to a perfect match was obtained (see Figure 3; more details on simulations are presented below).

Figure 4 shows the extinction spectra and TEM images of samples Number $2 \mathrm{a}$ and Number 1 at concentrations 0.5 and $0.1 \mathrm{~g} / \mathrm{dm}^{2}$, respectively. Those samples showed aging and precipitation after 1 week in water. Sample Number $2 \mathrm{a}$ had a narrower size distribution of the colloidal particles, while sample Number 1 had more size poly-dispersed colloidal nanoparticles. However, in both cases, the fragmentation in supercontinuum was not optimized for the most narrow size distribution, which as a rule, yielded in the most stable solutions. Water solutions of nanoparticles were mildly basic at $\mathrm{pH} 8.25$ (Number 2a) and 8.64 (Number 1). This is consistent with a negative charge of the gold-colloidal particles which attracts $\mathrm{H}^{+}$ions. This negative charging of the nanoparticles is the key reason for long-term stability of the colloidal solution and can be linked to the strong electron affinity to gold that was observed in organic solar cells. ${ }^{41}$ Exact mechanism of nanoparticle growth under laser irradiation needs further investigation. Usual growth predicted via microscopic solvability theory can be strongly violated at short time scales; ${ }^{42}$ also, a strong ionization ${ }^{43}$ of solution due to filamentation and white light continuum generation is expected to favor high zeta potential values required for stability of colloidal solution.

Sample Number 1 had nanoparticles with melt-like features whose shape was not spherical, while nanoparticles of sample

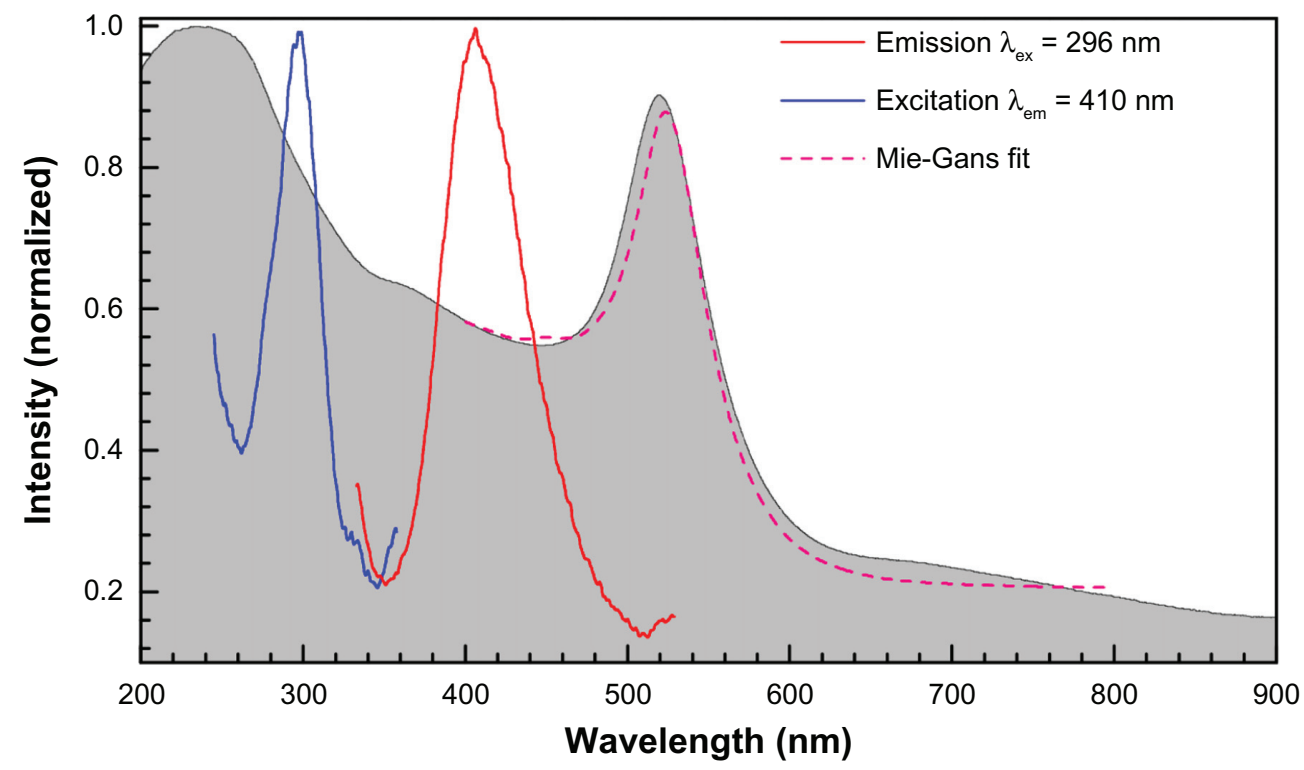

Figure 3 Normalized emission and experimental excitation spectra of the sample No 2 (shown in Figure 2) at the excitation wavelength $\lambda_{\text {ex }}=296$ nm and emission at $\lambda_{\mathrm{em}}=410 \mathrm{~nm}$, respectively; the normalized absorption spectrum is shown as a gray background. Theoretical fit by Mie-Gans theory based on Figure 2 data is shown. 

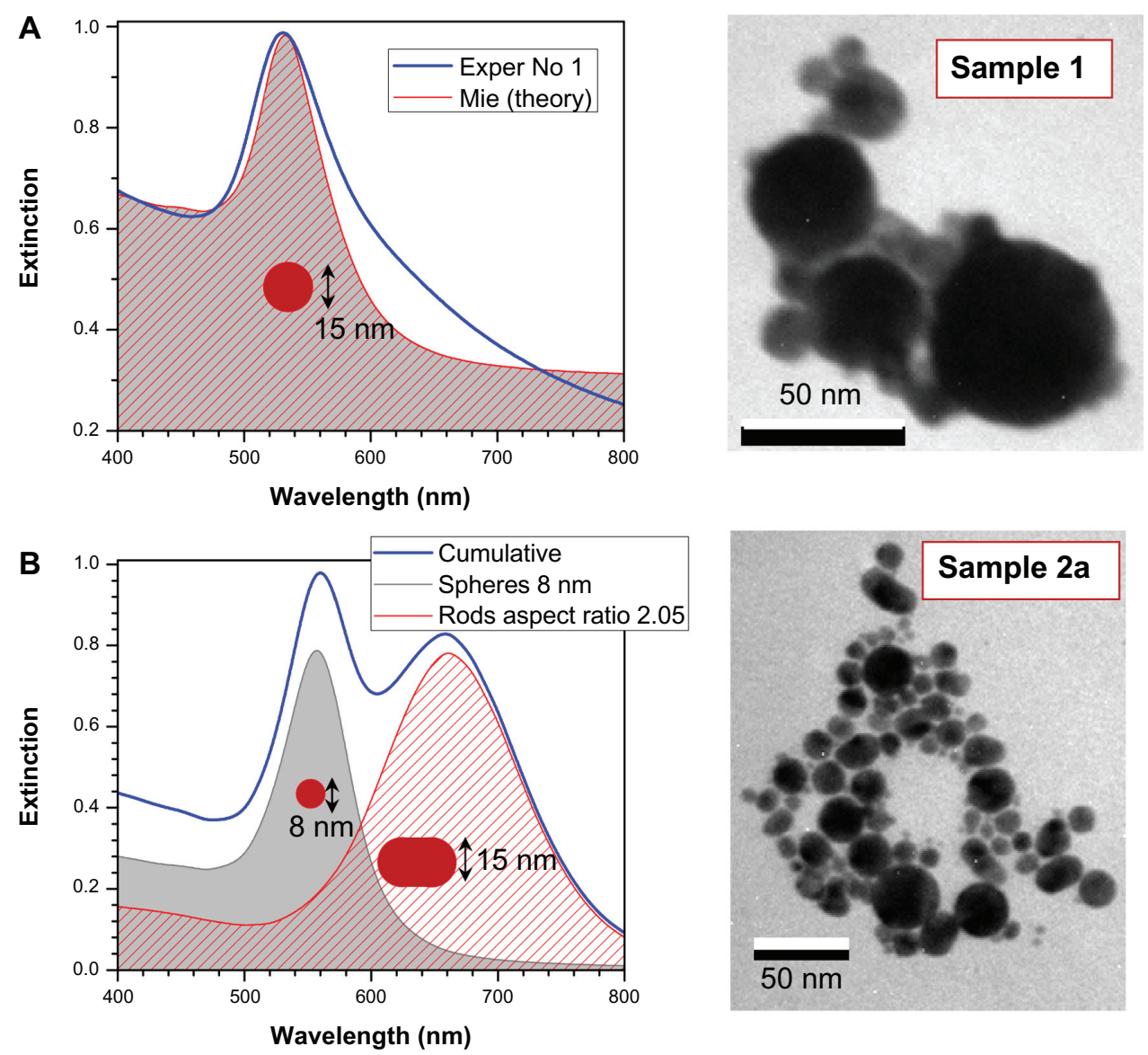

Figure 4 Simulated extinction spectra of less stable solutions No I (A) and No 2a (B) with representative transmission electron microscopy images. Assuming I cm cuvette length, the concentration of spheres was $9 \mathrm{nM}$ and rods $0.25 \mathrm{nM}$. The aspect ratio distribution of rods was $\sigma=0.5$. (A) Spectra of sample No I (Exper) fitted by: mean radius $15 \mathrm{~nm}$, standard deviation of radius $14 \mathrm{~nm}$, background correction baseline at 0.3 absorbance, total concentration of $530 \mathrm{nM}$.

Number $2 \mathrm{a}$ were spherical and elliptical with diameters ranging from 5 to $40 \mathrm{~nm}$ (the average diameter was approximately $15 \mathrm{~nm})$. It is possible to control to some extent the width of the size distribution of nanoparticles by choice of focusing, propagation length inside water, and pulse energy, which define supercontinuum generation. ${ }^{44}$ The optimized conditions were used to make stable solution Number 2 (Figure 3). It is noteworthy that a wider distribution of particle sizes is a promising feature for spectrally broadband sensing applications and might be useful for photo-catalysis and SERS.

The double feature in the extinction of sample Number $2 \mathrm{a}$ is consistent with elliptical particles (rod-shaped were used in modeling) of small aspect ratio $\sim 2$ recognizable in related TEM images. Fast thermal quenching of gold nanoparticles formed in the plume of ablation by femtosecond-laser pulses can capture elliptical nanoparticles before surface tension forms molten spheres. ${ }^{45}$ Figure 4 shows simulated extinction spectra for normally distributed spherical gold nanoparticles using full $\mathrm{Mie}^{46}$ theory and hemispherically capped cylindrical rods using electrostatically approximated
Mie-Gans ${ }^{47}$ theory; the mean and standard deviation of radius and aspect ratio are shown in the figure. A quantitative match is achievable when the size distribution is extracted from TEM observations. Sample Number 2a has spherical and rod-like particles of aspect ratio close to 2 (Figure 4B); sample Number 1 is dominated by spherical particles which have a large size distribution $d=15 \pm 14 \mathrm{~nm}$ (Figure 4A). The simulation fit in Figure 4A has been achieved by Mie theory. The shoulders at the longer wavelengths are due to the presence of elliptical and spherical nanoparticles, while at the shorter wavelength, a contribution of smaller few-nanometerdiameter nanoparticles is present. The smallest nanoparticles are absorptive and luminescent.

Samples Number 1 and 2 a were prone to agglomeration and showed changes consistent with the formation of larger particles as measured by optical extinction. Ultrasonic bath treatment revived the sample (the solution returned to a deep red color). An example of such behavior is illustrated in Figure 5 where the scattering spectrum is deconvoluted into the corresponding size distribution. (Note that the size distribution in Figure 3 was 

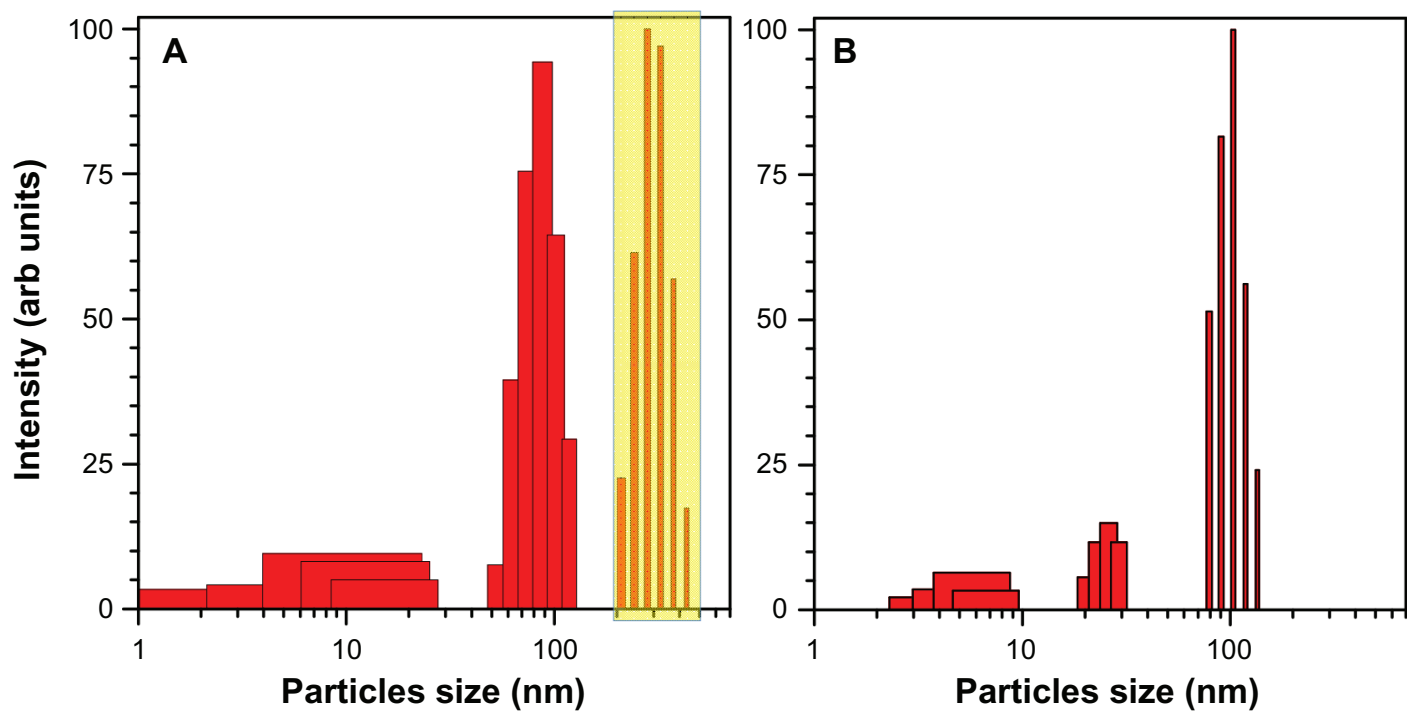

Figure $\mathbf{5}$ Ultrasonic revival of aged colloidal solution. (A) solution aged for $\sim$ I week and after ultrasonic agitation for 2 minutes. (B) The scattering intensity is normalized; the boxed region in $(\mathbf{A})$ shows particles that are formed by aging of the solution. Note that the abscissa axis is logarithmic. Sample solution Number $2 \mathrm{a}$.

judged by image processing and in Figure 4 by rigorous Mie scattering with consideration of shape and size effects.) The entire population of nanoparticles larger than $100 \mathrm{~nm}$ (assumed to be spherical) was wiped out by ultrasonic treatment.

\section{Fluorescence and its quenching}

The presence of an oxide cladding on the Au nanoparticles changes their chemical and optical properties. FLIM was used to determine a possible charge separation, plasmonic enhancement, and quenching. Fluorescence of gold ${ }^{48}$ has been detected using $392 \mathrm{~nm}$ excitation light. However, in our FLIM experiments we used a longer wavelength excitation. If the Au surface is covered with a dielectric cladding of up to several nanometers thickness, this can provide fluorescence enhancement of the dye around the nanoparticle (a pure metal surface usually quenches the fluorescence). Fluorescence of gold nanoparticles can also be excited by two-photon absorption. ${ }^{49}$ FLIM and SERS experiments were carried out to elucidate the issue of quenching.

To characterize the quenching of a common fluorescent dye (Rh6G) by Au nanoparticles with FLIM, the frequencydomain approach was utilized, wherein the lifetime is determined from the phase, $\phi$, and modulation, $M$, of the fluorescence signal. Figure 6 shows typical FLIM measurements on the phasor plot. The phasor plot is a convenient representation of the phase and modulation values, where:

$$
B=M \cos (\phi) \quad A=M \sin (\phi),
$$

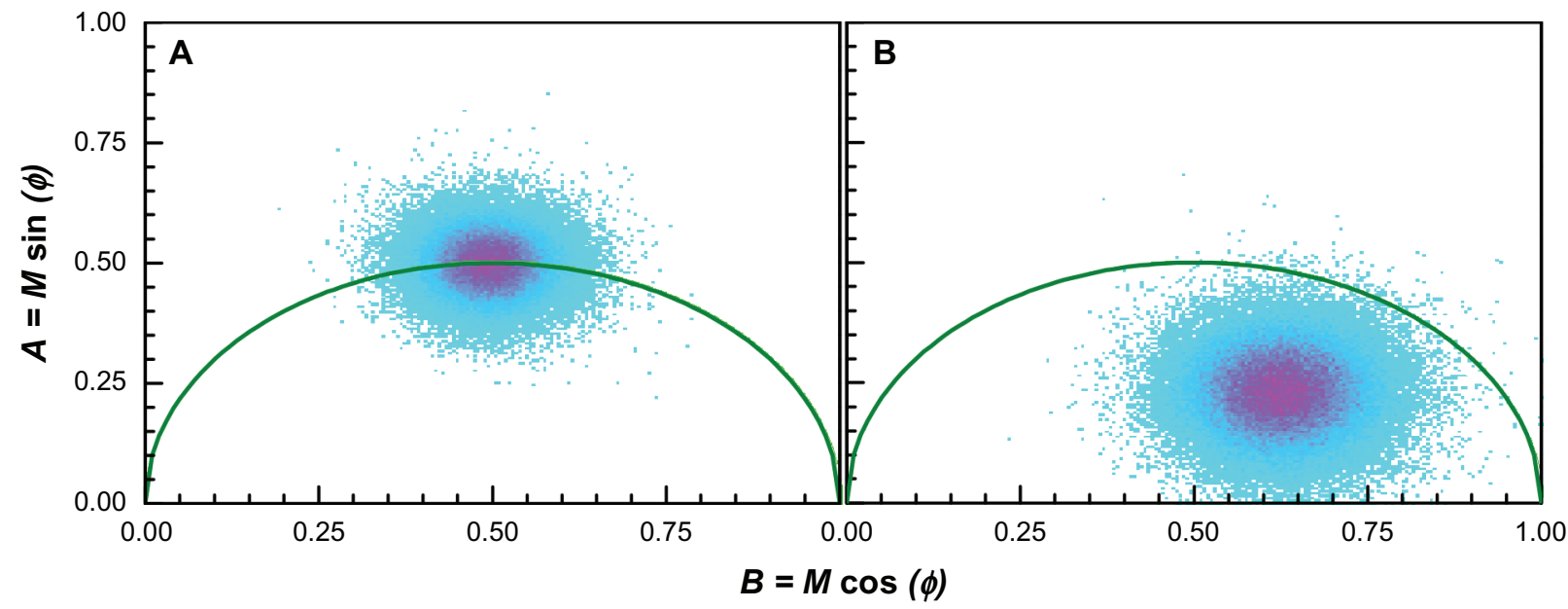

Figure 6 Phasor plots: (A) the fluorescence lifetime imaging microscopy phasor plot of rhodamine $6 \mathrm{G} 5 \mu \mathrm{M}$ and (B) sample No I with rhodamine $6 \mathrm{G} 5 \mu \mathrm{M}$ as a reference. 
Figure $6 \mathrm{~A}$ shows a phasor plot output for FLIM measurements of Rh6G $5 \mu \mathrm{M}$ where it is also used as a reference; Figure $6 \mathrm{~B}$ displays a lifetime measurement of sample Number 1 with the same concentration of Rh6G.

Comparing Figure 6A and B, a shift to the right (shorter lifetime) is evident, which would normally signify quenching of the rhodamine due to the presence of nanoparticles. However, because the concentration of fluorophore was low in our experiments, we wanted to exclude background fluorescence as a source of the apparent quenching. We therefore employed an approach first introduced by Caiolfa et $\mathrm{al}^{50}$ to remove the background signal. The idea is illustrated graphically in Figure 7. This approach involves first measuring the background phasor, then measuring the sample and background phasor, and then drawing a line through the two phasors and extrapolating to the semicircle. The intersection point with the semicircle contains information about the lifetime of the sample with the background removed. If we let the intersection point be at coordinates $(A, B)$ then the lifetime, $\tau$, is given by $\tau=\frac{A}{B \omega}$, where $\omega$ is the modulation frequency (for our setup, $\omega=2 \pi \times 40 \mathrm{MHz}$ or $0.25 \mathrm{~ns}^{-1}$ ).

The calculated lifetimes for Rh6G solution mixed with different concentrations of Number 2a and Number 1 samples are shown in Figure $8 \mathrm{~A}$ and $\mathrm{B}$, respectively. Turning first to sample Number 2a, Figure 8A, the lifetime of $12 \mathrm{nM} \mathrm{Rh6G}$ is $4.2 \mathrm{~ns}$ and apparently independent of gold nanoparticle concentration. For the $2.5 \mathrm{nM}$ Rh6G sample, a similar lifetime is observed; however, as the gold nanoparticle concentration exceeds 200 ppm, there is a progressive decrease in Rh6G lifetime. This is evidence that the gold nanoparticles are quenching the Rh6G solution. For sample Number 1, Figure 8B, the $12.5 \mathrm{nM}$ Rh6G solution appears to be weakly, if at all, quenched by the gold nanoparticles.

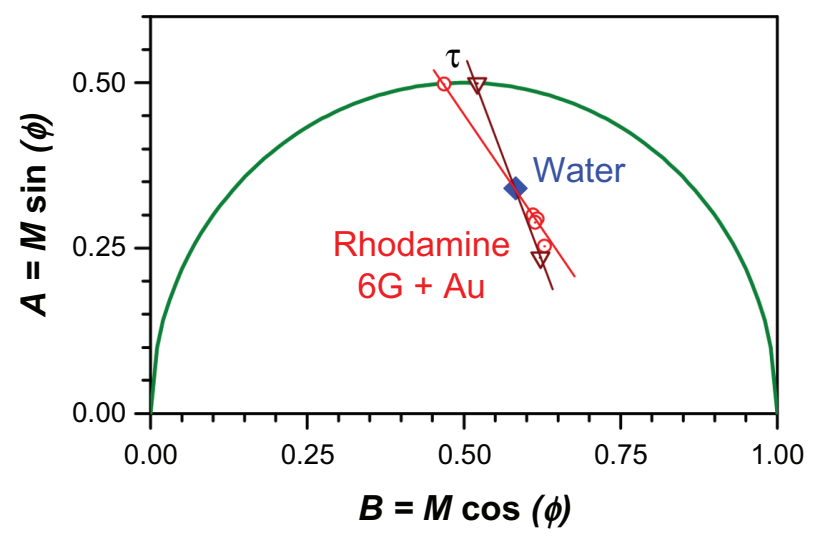

Figure 7 Determination of the fluorescence lifetime, $\tau=A /(B \omega)$, from a phasor plot, where $\omega$ is the modulation frequency; see text for details.
However, the $2.5 \mathrm{nM}$ Rh6G solution is strongly quenched (lifetime $\sim 2 \mathrm{~ns}$ ) by the nanoparticles at all concentrations tested (20-100 ppm range).

Fluorescence quenching is consistent with the pure-metallic nature of Au nanoparticles ${ }^{51}$ obtained by ablation in water and is discussed further in the Discussion section.

\section{SERS}

The SERS spectrum of diluted Rh6G solution $(4.3 \mu \mathrm{M})$ was measured using Au nanoparticles (solution Number 1 after ultrasonic resuspension) attached to a silicon wafer surface by a mercaptosilane cross linker. The immobilized Au nanoparticles were expected to act as an SERS substrate. ${ }^{52}$ When the nanoparticles were exposed to the Rh6G solution, the SERS spectrum clearly showed the Rh6G signature peaks with a small shift from those in solution ${ }^{53}$ at: 611 (614 in solution), 773 (774), 1124 (1129), 1185 (1183), 1312 (1310), 1364 (1363), 1506 (1509), and 1645 (1650) as shown in Figure 9A. Change of spectral positions of the peaks by $\pm(7-10) \mathrm{cm}^{-1}$ corresponds to $1.24 \mathrm{meV}$ or $0.12 \mathrm{~kJ} / \mathrm{mol}$ and is expected for adsorbed molecules. The differential spectrum (Figure 9B) clearly reveals Rh6G and demonstrates the feasibility of using laser-ablated Au nanoparticles for SERS sensing.

\section{Discussion}

For conventional dynamic quenching of fluorophores, the lifetime of fluorescence should decrease with increasing quencher concentration and also the quenching should be independent of fluorophore concentration. For static quenching, which occurs through complex formation, the lifetime is usually not affected, but the static quenching can depend on the stoichiometry of the fluorophore and quencher.

The results obtained with the Rh6G and gold nanoparticles appear to be intermediate between a static and a dynamic quenching mechanism. We propose that owing to the electrostatics on the gold particles, Rh6G is attracted to the gold particle surface. This is consistent with an electron affinity of gold, which for example, causes a charge separation in organic solar cells. ${ }^{41}$ The large size of the gold nanoparticles enables many Rh6G molecules to bind, which are then effectively quenched by a short range mechanism, perhaps electron transfer. ${ }^{54}$ This sort of fluorescence quenching process is well known for Rh6G on silver nanoparticles, where it plays an important role in enabling single molecule spectroscopy ${ }^{5}$ and is likely to play a similar role in gold nanoparticles. ${ }^{55}$ Evidence for electron transfer is readily seen at the low concentration of Rh6G, where there should be a high proportion of bound Rh6G in complex with the nanoparticles. But when the Rh6G 


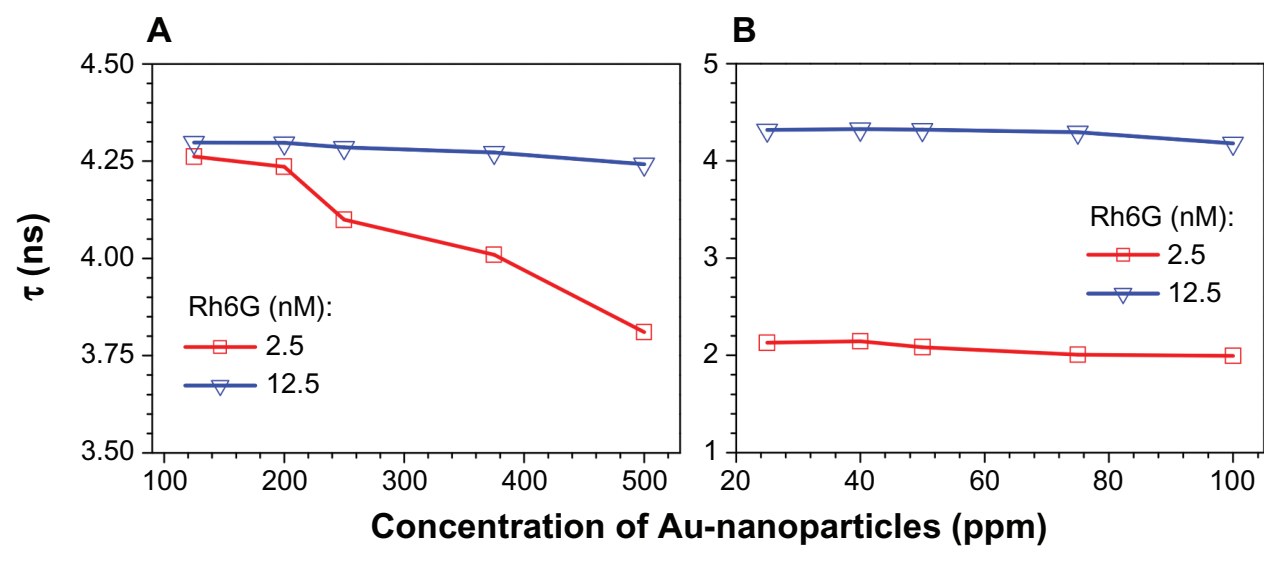

Figure 8 Lifetime, $\tau$, versus concentration of gold nanoparticles in Rh6G at two different dye concentrations for samples No $2 a(\mathbf{A})$ and No I (B). Abbreviation: Rh6G, rhodamine 6G.

concentration is increased, the bound Rh6G molecules are already saturated on the gold surfaces, and the proportion of free Rh6G increases and effectively dominates the total fluorescence signal. In this case, the normal lifetime of Rh6G is observed and is independent of nanoparticle concentration.
The different behavior of the Number $2 \mathrm{a}$ and Number 1 particles can be accounted for in this mechanism by the different surface availabilities for Rh6G.

The initially untreated Au nanoparticles are a promising platform for surface functionalization and attachment to

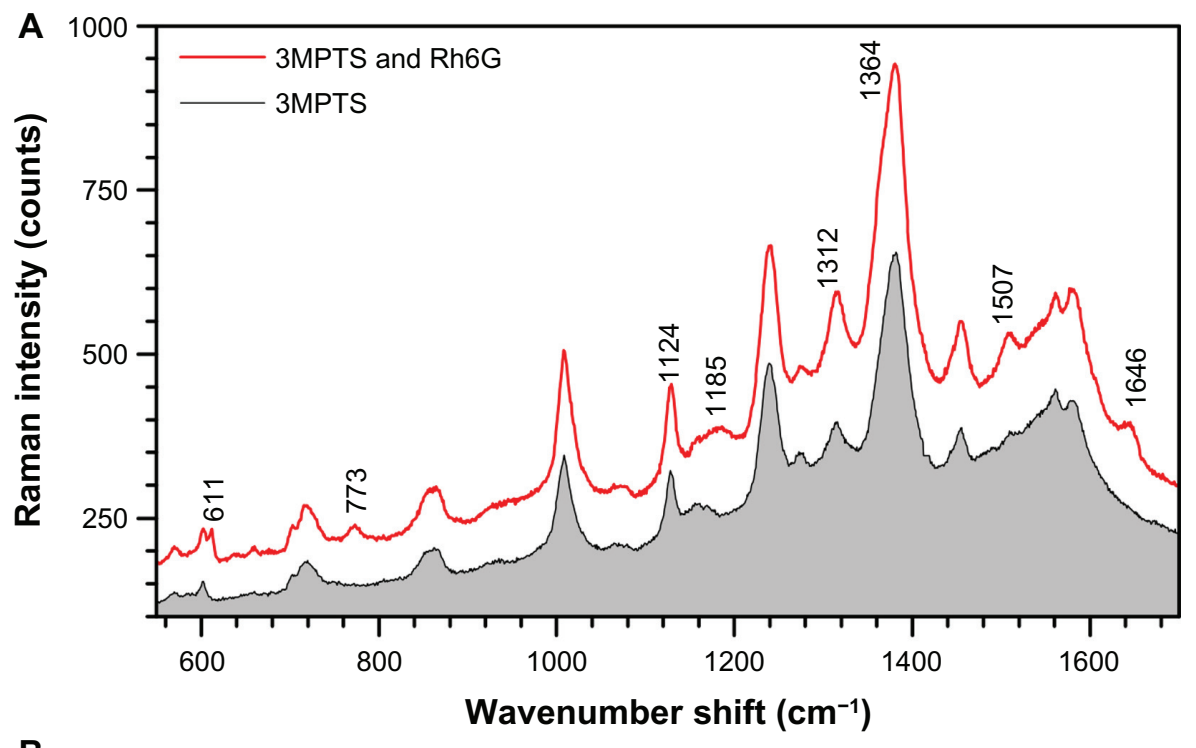

B

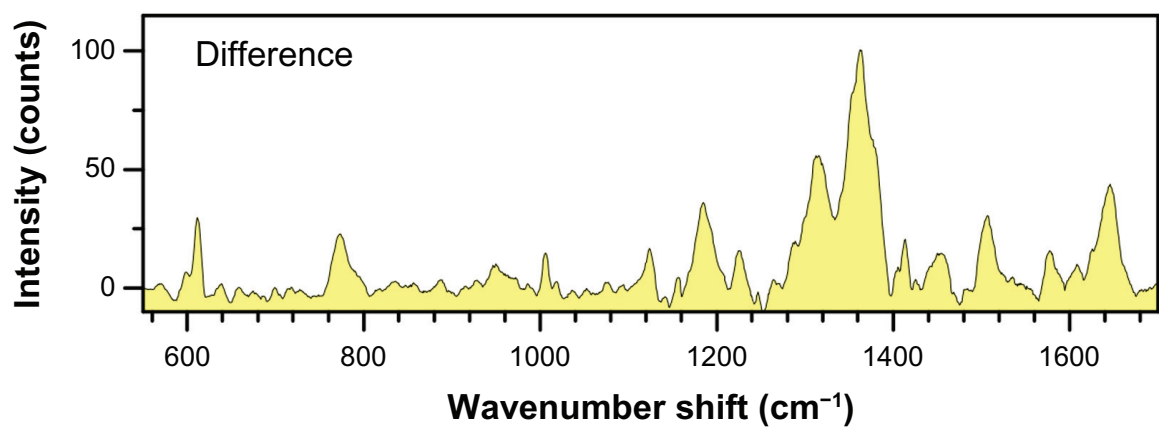

Figure 9 (A) Comparison of surface-enhanced Raman spectroscopy spectra from gold (Au)-nanoparticles (solution No I) treated with mercaptosilane cross linker and with Rh6G. (B) The same sample showing peaks for $4.3 \mu \mathrm{M}$ Rh6G after subtracting the mercaptosilane background.

Abbreviations: 3MPTS, (3-mercaptopropyl) trimethoxysilane; Rh6G, rhodamine 6G. 
other surfaces. Importantly, aqueous systems that are vital for biological and medical applications are directly compatible with Au nanoparticles formed by ablation in deionized water. In contrast, chemically synthesized gold nanoparticles require substantial modification before they are compatible with these applications. ${ }^{56}$ Stable colloidal solutions will find applications in microfluidic and laser trapping fields $s^{57,58}$ and could be used on nanotextured sensing platforms such as black silicon. ${ }^{59}$ New pathways to make chemical bonding of nanoparticles are expected to find applications in imaging via plasmonic light scattering inside cells and for medical applications. ${ }^{17-19}$

\section{Conclusion}

A facile method of fabricating stable gold nanoparticles of controlled size and shape distribution by femtosecond-laser ablation and fragmentation in water is demonstrated. The high concentration solutions obtained via this process were characterized optically, structurally, and their fluorescence quenching behavior has been revealed.

Laser produced Au nanoparticles can be centrifuged for even better size separation. New pathways of chemical functionalization of such nanoparticles can be envisaged as compared with chemically synthesized and stabilized Au colloids. SERS applications will benefit from a wider choice of available surface modifications compatible with aqueous solutions in biological and medical applications.

\section{Acknowledgments}

RK acknowledges support via a fellowship funded by European Union Structural Funds project "Postdoctoral Fellowship Implementation in Lithuania" within the framework of the Measure for Enhancing Mobility of Scholars and Other Researchers and the Promotion of Student Research (VP1-3.1-ŠMM-01) of the Program of Human Resources Development Action Plan. The project was in part supported by the Australian Research Council DP120102980 project, VP1-3.1-ŠMM-01-V-02-001 funding scheme, and French National Research Agency (ANR) grants LASERNANOBIO (ANR-10-BLAN-919) and BIONANOPLASMON (ANR09-BLAN-0177).

\section{Disclosure}

The authors report no conflicts of interest in this work.

\section{References}

1. Sokolov K, Follen M, Aaron J, et al. Real-time vital optical imaging of precancer using anti-epidermal growth factor receptor antibodies conjugated to gold nanoparticles. Cancer Res. 2003;63:1999-2004.
2. Hirsch LR, Stafford RJ, Bankson JA, et al. Nanoshell-mediated near-infrared thermal therapy of tumors under magnetic resonance guidance. Proc Nat Acad Sci. 2003;100:13549-13554.

3. Huang X, El-Sayed IH, Qian W, El-Sayed MA. Cancer cell imaging and photothermal therapy in near-infrared region by using gold nanorods. J Am Chem Soc. 2006;128:2115-2120.

4. Sobhan MA, Sreenivasan VKA, Withford MJ, Goldys EM. Non-specific internalization of laser ablated pure gold nanoparticles in pancreatic tumor cell. Colloids Surf B Biointerfaces. 2012;92:190-195.

5. Nie S, Emory SR. Probing single molecules and single nanoparticles by surface-enhanced Raman scattering. Science. 1997;275: 1102-1106.

6. Kneipp K. Surface-enhanced Raman scattering. Phys Today. 2007; 60:40-47.

7. White IM, Yazdi SH, Yu WW. Optofluidic SERS: synergizing photonics and microfluidics for chemical and biological analysis. Microfluid Nanofluid. 2012;13(2):205-216.

8. Qian X, Peng XH, Ansari DO, et al. In vivo tumor targeting and spectroscopic detection with surface-enhanced Raman nanoparticle tags. Nat Biotechnol. 2008;26:83-90.

9. Lin CAJ, Lee CH, Hsieh JT, et al. Synthesis of fluorescent metallic nanoclusters toward biomedical application: Recent progress and present challenges. J Med Biol Eng. 2009;29(6):276-283.

10. Wilcoxon JP, Martin JE, Parsapour F, Wiedenman B, Kelley DF. Photoluminescence from nanosize gold clusters. J Chem Phys. 1998;108:9137.

11. Williams CG, Malik AN, Kim TK, Manson PN, Elisseeff JH. Variable cytocompatibility of six cell lines with photoinitiators used for polymerizing hydrogels and cell encapsulation. Biomaterials. 2005;26:1211-1218.

12. Albrecht AM, Evans CW, Raston CL. Green chemistry and the health implications of nanoparticles. Green Chem. 2006;80:417-432.

13. Elavazhagan T, Arunachalam KD. Memecylon edule leaf extract mediated green synthesis of silver and gold nanoparticles. Int $J$ Nanomedicine. 2011;6:1265-1278.

14. Zhang YX, Zheng J, Gao G, et al. Biosynthesis of gold nanoparticles using chloroplasts. Int J Nanomedicine. 2011;6:2899-2906.

15. Letfullin RR, George TF, Duree GC Jr, Bollinger B. Ultrashort laser pulse heating of nanoparticles: comparison of theoretical approaches, advances in optical technologies. Advances in Optical Technologies. 2008;2008:Article ID 251718.

16. Letfullin RR, Joenathan C, George TF, Zharov VP. Cancer cell killing by laser-induced thermal explosion of nanoparticles. J Nanomedicine. 2006;1(4):473-480.

17. Kho KW, Fu CY, Dinish US, Olivo M. Clinical SERS: are we there yet? J Biophotonics. 2011;4(10):667-684.

18. Lozovski V, Lysenko V, Piatnytsia V, Scherbakov O, Zholobak N, Spivak M. Physical point of view for antiviral effect caused by the interaction between the viruses and nanoparticles. J Bionanoscience. 2012;6:109-112.

19. Letfullin RR, Rice CEW, George TF. Theoretical study of bone cancer therapy by plasmonic nanoparticles. Ther Deliv. 2011;2(10): $1259-1273$.

20. Kabashin AV, Delaporte P, Pereira A, Grojo D, Torres D, Sarnet T, Sentis M. Nanofabrication with pulsed lasers. Nanoscale Res Lett. 2010; 5(3):454-463.

21. Fojtik A, Henglein A. Laser ablation of films and suspended particles in a solvent: formation of cluster and colloid solutions. Ber Bunsenges Phys Chem. 1993;97:252-254.

22. Sibbald MS, Chumanov G, Cotton TM. Reduction of cytochrome c by halide-modified, laser-ablated silver colloids. J Phys Chem C. 1996;100:4672-4678.

23. Mafune F, Kohno J-Y, Takeda Y, Kondow T, Sawabe H. Formation of gold nanoparticles by laser ablation in aqueous solution of surfactant. J Phys Chem B. 2000;104:9111-9117.

24. Mafune F, Kohno J-Y, Takeda Y, Kondow T, Sawabe H. Formation of gold nanopaticles by laser ablation in aqueous solution of surfactant. J Phys Chem B. 2001;105:5114-5120. 
25. Dolgaev SI, Simakin AV, Voronov VV, Shafeev GA, Bozon-Verduraz F. Nanoparticles produced by laser ablation of solids in liquid environment. Appl Surf Sci. 2002;186:546-551.

26. Tsuji T, Iryo K, Watanabe N, Tsuji M. Preparation of silver nanoparticles by laser ablation in solution: influence of laser wavelength on particle size. Appl Surf Sci. 2002;202:80-85.

27. Desarkar HS, Kumbhakar P, Mitra AK. Linear optical absorption and photoluminescence emission properties of gold nanoparticles prepared by laser ablation technique. Appl Phys A. 2012;108(1):81-89.

28. Pyatenko A, Yamaguchi M, Suzuki M. Mechanisms of size reduction of colloidal silver and gold nanoparticles irradiated by Nd:YAG laser. J Phys Chem C. 2009;113:9078-9085.

29. Kabashin AV, Meunier M. Synthesis of colloidal nanoparticles during femtosecond laser ablation of gold in water. J Appl Phys. 2003;94: 7941-7943.

30. Kabashin AV, Meunier M. Femtosecond laser ablation in aqueous solutions: a novel method to synthesize non-toxic metal colloids with controllable size. J Phys Conf Series. 2006;59:354-357.

31. Sylvestre J-P, Poulin S, Kabashin AV, Sacher E, Meunier M, Luong JHT. Surface chemistry of gold nanoparticles produced by laser ablation in aqueous media. J Phys Chem B. 2004;108:16864-16869.

32. Kabashin AV, Meunier M, Kingston C, Luong JHT. Fabrication and characterization of gold nanoparticles by femtosecond laser ablation in aqueous solution of cyclodextrins. J Phys Chem B. 2003;107: 4527-4531.

33. Besner S, Kabashin AV, Winnik FM, Meunier M. Synthesis of size-tunable polymer-protected gold nanoparticles by femtosecond laser-based ablation and seed growth. J Phys Chem C. 2009;113:9526-9531.

34. Besner S, Kabashin AV, Meunier M. Two-step femtosecond laser ablation-based method for the synthesis of stable and ultra-pure gold nanoparticles in water. Appl Phys A. 2007;88:269-272.

35. Videla FA, Torchia GA, Schinca DC, et al. Analysis of the main optical mechanisms responsible for fragmentation of gold nanoparticles by femtosecond laser radiation. J Appl Phys. 2010;107:114308.

36. Besner S, Kabashin AV, Meunier M. Fragmentation of colloidal nanoparticles by femtosecond laser-induced super continuum generation. Appl Phys Lett. 2006;89:233121-233123.

37. Liu W, Kosareva OG, Golubtsov IS, et al. Femtosecond laser pulse filamentation versus optical breakdown in $\mathrm{H}_{2} \mathrm{O}$. Appl Phys B. 2003;76: 215-229.

38. Vu TKT, Nguyen QD, Nguyen TD, Trinh TH. Preparation of metal nanoparticles for surface enhanced Raman scattering by laser ablation method. Adv Nat Sci Nanosci Nanotechnol. 2012;3:025016.

39. Stockman MI. Nanoplasmonics: past, present, and glimpse into future Optics Express. 2011;19(22):22029-22106.

40. Boyd GT, Yu ZH, Shen YR. Photoinduced luminescence from the noble metals and its enhancement on roughened surfaces. Phys Rev B. 1986;33:7923-7936

41. Poh CH, Rosa L, Juodkazis S, Dastoor P. FDTD modeling to enhance the performance of an organic solar cell embedded with gold nanoparticles. Opt Mater Express. 2011;1:1326-1331.
42. Basu H, Kolwankar KM, DharmadhikariAK, etal. Laser-driven accelerated growth of dendritic patterns in liquids. J Phys Chem C. 2012;116(21): 11480-11485.

43. Rajgara FA, Dharmadhikari AK, Mathur D, Safvan CP. Strong fields induce ultrafast rearrangement of $\mathrm{H}$ atoms in $\mathrm{H}_{2} \mathrm{O}$. J Chem Phys. 2009;130:231104

44. Kabashin AV. Laser ablation-based methods for nanostructuring of materials. Laser Physics. 2009;19(5):1136-1141.

45. Juodkazis S, Misawa H, Louchev OA, Kitamura K. Femtosecond laser ablation of chalcogenide glass: explosive formation of nano-fibers against thermo-capillary growth of micro-spheres. Nanotechnology. 2006;17:4802-4805

46. Mie G. Beiträge zur optik trüber medien, speziell kolloidaler metallösungen. [Contributions to the optics of turbid media especially colloidal metal suspensions]. Ann Phys. 1908:25:377-455 German.

47. Gans R. Über die form ultramikroskopischer goldteilchen. [On the form of ultra microscopic gold particles]. Ann Phys. 1912;342(5):881-900 German.

48. Mooradian A. Photoluminescence of metals. Phys Rev Lett. 1969;22(5): 185-187.

49. Ueno K, Juodkazis S, Mizeikis V, Sasaki K, Misawa H. Clusters of closely spaced gold nanoparticles as a source of two-photon photoluminescence at visible wavelengths. Adv Mat. 2008;20: 26-29.

50. Caiolfa VR, Zamai M, Malengo G, et al. Monomer dimer dynamics and distribution of GPI-anchored uPAR are determined by cell surface protein assemblies. J Cell Biol. 2007;179(5):1067-1082.

51. Cook KM, Ferguson GS. Relative lability of gold-oxide thin films in contact with air, solvents, or electrolyte solutions. J Vac Sci Technol A. 2013;31:021508

52. Polwart E, Keir RL, Davidson CM, Smith WE, Sadler DA. Novel SERS-active optical fibers prepared by the immobilization of silver colloidal particles. Appl Spectrosc. 2000;54:522-527.

53. Hildebrandt P, Stockburger M. Surface-enhanced resonance Raman spectroscopy of rhodamine 6G adsorbed on colloidal silver. $J$ Phys Chem. 1984;88(24):5935-5944.

54. Thomas KG, Kamat PV. Chromophore-functionalized gold nanoparticles. Acc Chem Res. 2003;36(12):888-898.

55. Kneipp K, Kneipp H, Manoharan R, et al. Extremely large enhancement factors in surface-enhanced Raman scattering for molecules on colloidal gold clusters. Appl Spectrosc. 1998;52(12):1493-1497.

56. Kneipp J, Li X, Sherwood M, Panne U, et al. Gold nanolenses generated by laser ablation-efficient enhancing structure for surface. 2008;80:4247-4251.

57. Ivanova EP, Truong VK, Gervinskas G, et al. Highly selective trapping of enteropathogenic E. coli on Fabry-Perot sensor mirrors. Biosens Bioelectron. 2012;35:369-375.

58. Misawa H, Juodkazis S. Photophysics and photochemistry of a laser manipulated microparticle. Prog Polym Sci. 1999;24:665-697.

59. Žukauskas A, Malinauskas M, Kadys A, et al. Black silicon: substrate for laser 3D micro/nano-polymerization. Optics Express. 2013:21: 6901-6909.
International Journal of Nanomedicine

\section{Publish your work in this journal}

The International Journal of Nanomedicine is an international, peerreviewed journal focusing on the application of nanotechnology in diagnostics, therapeutics, and drug delivery systems throughou the biomedical field. This journal is indexed on PubMed Central,

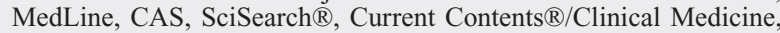

\section{Dovepress}

Journal Citation Reports/Science Edition, EMBase, Scopus and the Elsevier Bibliographic databases. The manuscript management system is completely online and includes a very quick and fair peer-review system, which is all easy to use. Visit http://www.dovepress.com/ testimonials.php to read real quotes from published authors. 